\title{
HIGHLIGHTS
}

GENETICS

\section{Duplication in CFHR5: a new culprit in inherited renal disease}

An internal duplication within the gene that encodes complement factor-H-related protein 5 (CFHR5) is responsible for causing renal disease in individuals of Cypriot origin, say researchers. "We think this is the first clear example of such a change causing a highly penetrant disease", explains one of the study's coauthors, Patrick Maxwell.

To identify new genetic causes of renal disease, Maxwell and colleagues initially performed genome-wide linkage analysis in two families from a London renal transplant center. The families were both of Cypriot origin and had family members who were affected by unusual renal disease that seemed to show an autosomal dominant mode of inheritance. The researchers identified a haplotype within chromosome 1q31-32 that cosegregated with the occurrence of disease. "We had two pedigrees with a very rare, very similar condition, who were originally from the same country. We reasoned that they were likely to have the same genetic problem, and this turned out to be the case", states Maxwell.

The researchers then used a candidate gene approach to identify a novel variant that resulted in duplication of exons 2 and 3 of CFHR5 in affected individuals. A PCRbased diagnostic test, developed by the researchers, was used to demonstrate the presence of the mutation in additional individuals from Cyprus or of Cypriot origin with unexplained hematuria or with end-stage renal disease of unknown cause. By contrast, the researchers were unable to detect the CFHR5 duplication in any of 36 individuals tested from the UK or France who had biopsy samples showing C3 glomerulonephritis.

Maxwell and colleagues say that their findings imply that CFHR5 may be crucial in protecting the normal kidney from immunological attack. They say that their

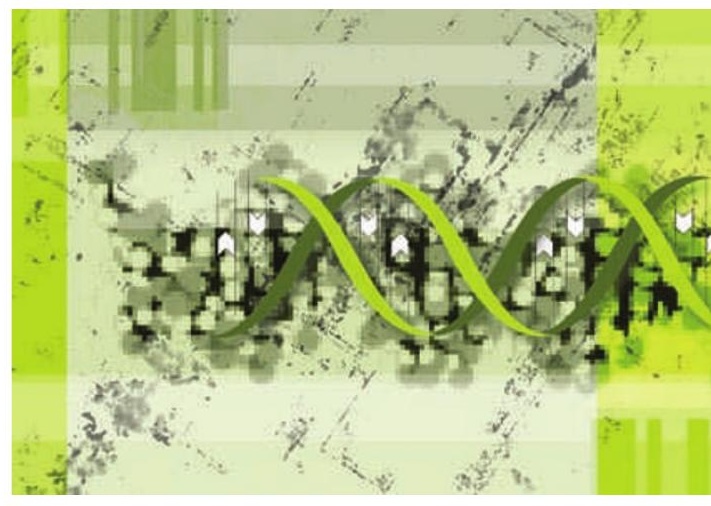

findings provide evidence of an inherited renal disease, which they term CFHR5 nephropathy, charcterized by hematuria, that may account for a substantial proportion of renal disease affecting Cypriots and their descendants worldwide.

Susan J. Allison

Original article Gale, D. P. et al. Identification of a mutation in complement factor H-related protein 5 in patients of Cypriot origin with glomerulonephritis. Lancet 376 794-801 (2010)

Further reading Karumanchi, S. A. \& Thadhani, R. A complement to kidney disease: CFHR5 nephropathy. Lancet 376, 748-750 (2010) 